\title{
ОПРЕДЕЛЕНИЕ ФРАКЦИОННОГО СОСТАВА СЛАНЦЕВЫХ СМОЛ МЕТОДОМ ИМИТИРОВАННОЙ ДИСТИЛЛЯЦИИ
}

В последние годы стало актуальным исследование сланцев с точки зрения органической геохимии. С целью характеристики исходного органического вещества сланцы в лабораторных условиях подвергают переработке различными методами, например, полукоксованию в реторте Фишера или деструктивной гидрогенизации в автоклаве, причем получаются смолы, количество которых варьирует от 1 до 20 г в зависимости от природы сланца. Определить фракционный состав смолы, пользуясь обычными методами, как, например, ректификацией или дистилляцией по Энглеру, из-за малого количества пробы не представляется возможным. Да и вообще получение кривых разгонки высококипящих продуктов названными методами практически невозможно. Нередко при исследовании необходимо оценить также количество нехроматографи. руемой части смолы.

Перспективным по получению кривых разгонки лабораторных и промышленных сланцевых продуктов является хроматографический метод имитированной дистилляции, который состоит в разделении смеси в короткой колонке в условиях программирования температуры. Выходящее из колонки количество вещества регистрируется с помощью интегратора. Линейная зависимость, существующая между временем

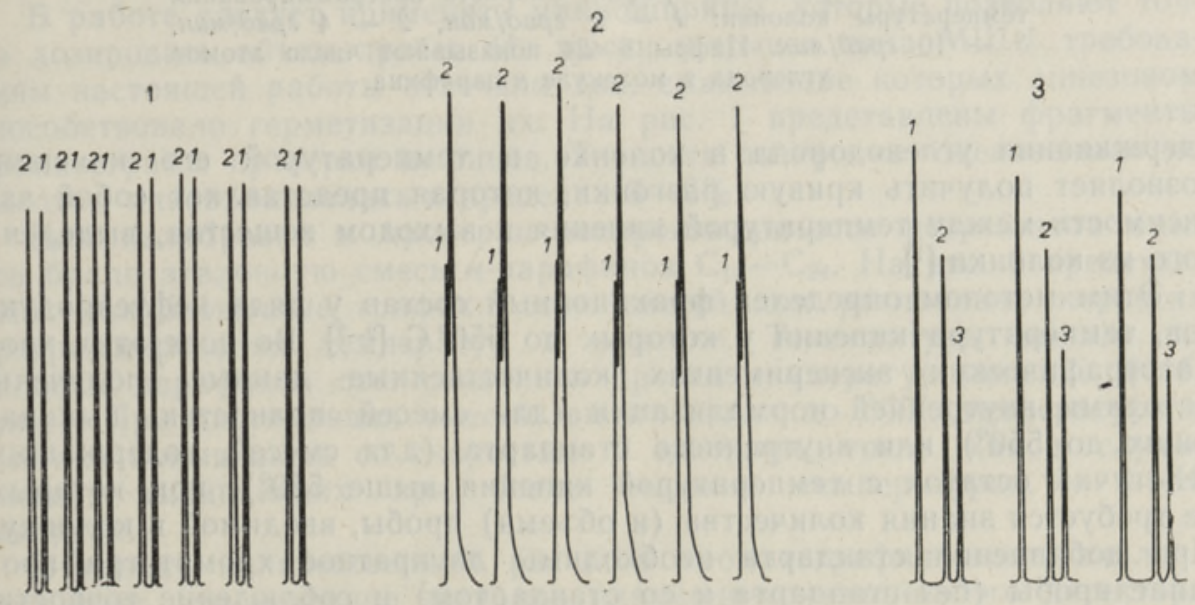

Рис. 1. Фрагменты хроматограмм, полученных от проверки микрошприцев. В изотермическом режиме: 1 - шприц МШ̈-І, объем пробы 0,8 мкл; 2 - шприц фирмы «Гамильтон», объем пробы 0,6 мкл. При скорости программирования температуры колонки 10 град/мин: 3 - шприц МШ-І, объем пробы 1,0 мкл. Цифры на пиках показывают порядок выхода компонентов из колонки. 


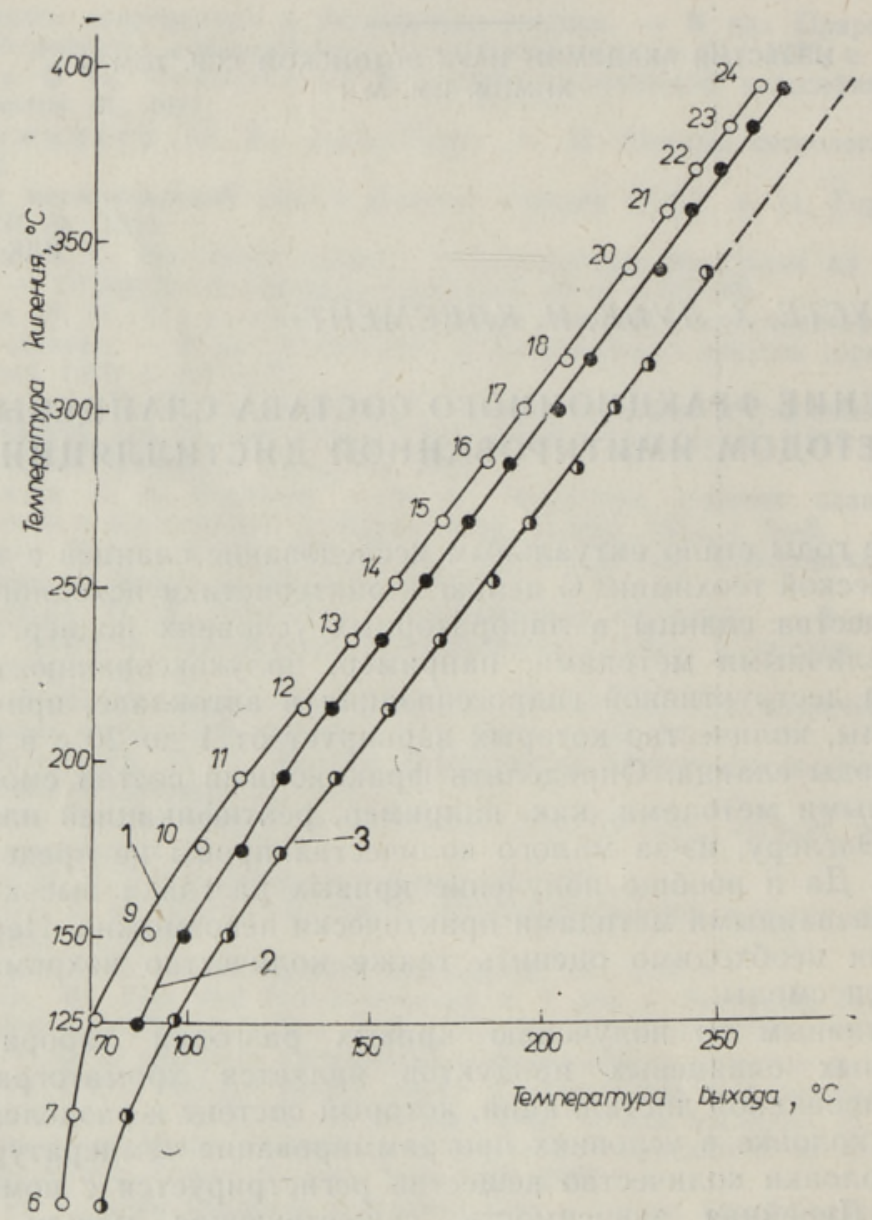

Рис. 2. Калибровочный график. Скорость программирования температуры колонки: $1-2$ град/мин, $2-4$ град/мин, 3 - 10 град/мин. Цифры 6-24 показывают число атомов углерода в молекуле $\boldsymbol{H}$-парафина.

удерживания углеводорода в колонке и температурой его кипения, позволяет получить кривую разгонки, которая представляет собой зависимость между температурой кипения и выходом вещества, выделенного из колонки ['].

Этим методом определен фракционный состав у ряда нефтепродуктов, температура кипения у которых до $550^{\circ} \mathrm{C}\left[{ }^{2-7}\right]$. Во всех этих хроматографических экспериментах количественные данные получены методами внутренней нормализации (для смесей полностью выкипающих до $550^{\circ}$ ) или внутреннего стандарта (для смесей, содержащих нелетучий остаток с температурой кипения выше $550^{\circ}$ ), при которых не требуется знания количества (и объема) пробы, вводимой в колонку. При добавлении стандарта необходимы двукратное хроматографиро. вание пробы (без стандарта и со стандартом) и соблюдение точности взвешивания малых количеств вещества.

Представляет интерес разработать простой метод имитированной дистилляции, который основывается на точном объеме пробы и удельном весе ее. При использовании такого метода расход пробы минимален, и в то же время отпадают все трудности, связанные с взвешива- 
Результаты имитированной дистилляции эталонной смеси $\boldsymbol{H}$-парафинов $\mathrm{C}_{10}-\mathrm{C}_{24}$

\begin{tabular}{|c|c|c|c|c|c|}
\hline \multirow[b]{2}{*}{$\begin{array}{l}\text { Компо- } \\
\text { нент }\end{array}$} & \multirow[b]{2}{*}{$\begin{array}{l}\text { Темпера- } \\
\text { тура ки- } \\
\text { пения, }{ }^{\circ} \mathrm{C}\end{array}$} & \multirow[b]{2}{*}{$\begin{array}{l}\text { Взято, } \\
\text { вес. } \%\end{array}$} & \multirow[b]{2}{*}{$\begin{array}{c}\text { Получено, } \\
\text { вес. } \%\end{array}$} & \multicolumn{2}{|c|}{ Отклонение, \% } \\
\hline & & & & абсолютное & $\begin{array}{l}\text { относн- } \\
\text { тельное }\end{array}$ \\
\hline $\mathrm{C}_{10}$ & 174,0 & 10,1 & 9,6 & $-0,5$ & 5,0 \\
\hline $\mathrm{C}_{11}$ & 195,8 & 10,7 & 10,9 & $+0,2$ & $+1,9$ \\
\hline $\mathrm{C}_{12}$ & 216,2 & 9,5 & 9,9 & +0.4 & $+4,2$ \\
\hline $\mathrm{C}_{13}$ & 235,4 & 8,0 & 7,9 & $-0,1$ & $+1,2$ \\
\hline $\mathrm{C}_{14}$ & 253,6 & 9,4 & 9,8 & $+0,4$ & $+4,3$ \\
\hline $\mathrm{C}_{15}$ & 270,6 & 9,2 & 9,2 & 0 & 0 \\
\hline $\mathrm{C}_{16}$ & 287,1 & 8,9 & 8,7 & $-0,2$ & $-2,5$ \\
\hline $\mathrm{C}_{17}$ & 302,7 & 9,5 & 9,9 & $+0,4$ & $+4,2$ \\
\hline $\mathrm{C}_{18}$ & 316,1 & 6,4 & 6,1 & $-0,3$ & $-4,7$ \\
\hline $\mathrm{C}_{19}$ & 330,0 & - & - & - & - \\
\hline $\mathrm{C}_{20}$ & 342,7 & 4,3 & 4,1 & $-0,2$ & $-4,8$ \\
\hline $\mathrm{C}_{2 !}$ & 358,4 & 4,4 & 4,5 & $+0,1$ & $+2,3$ \\
\hline $\mathrm{C}_{22}$ & 370,0 & 3,0 & 2,9 & $-0,1$ & $-3,3$ \\
\hline $\mathrm{C}_{23}$ & 382,0 & 5,0 & 4,9 & $-0,1$ & $-2,0$ \\
\hline $\mathrm{C}_{24}$ & 394,0 & 1,6 & 1,6 & 0 & 0 \\
\hline
\end{tabular}

нием малых количеств проб, а также необходимость в двукратном хроматографировании, что значительно сокращает время анализа.

В настоящей работе хроматографирование исследуемых проб проводилось на газовом хроматографе «Хром-41», снабженном интегратором. Детектор - пламенно-ионизационный. Колонка: 3\% OV 101 на хроматоне $N$-AW $H M D S, 1,5 \mu \times 3 м$. Температура термостата программировалась в диапазоне $50-320^{\circ}$ со скоростью $2-10$ град/мин. Расход газа-носителя гелия составлял $20 \mathrm{~m} /$ мин. Температура в испарителе поддерживалась на уровне $330-350^{\circ}$. Для ввода проб использовали шприцы типа МШ-1 и фирмы «Гамильтон» объемом в 1 мкл; объем вводимой пробы 0,8 мкл. Для компенсации дрейфа нулевой линии применяли систему параллельных колонок. Әффективность колонки по н-декану при $100^{\circ}$ составляла 1560 тт.

В работе следует применять микрошприцы, которые позволяют точно дозировать объем пробы. Из шести шприцев типа MШ-1 требованиям настоящей работы отвечали два, смазывание которых апиезоном способствовало герметизации их. На рис. 1 представлены фрагменты хроматограмм проверки шприцев. Точность дозировки, оцениваемая по высотам пиков, оказалась в пределах $3-6 \%$.

Для калибровки и проверки воспроизводимости результатов опытов брали эталонную смесь $н$-парафинов $\mathrm{C}_{10}-\mathrm{C}_{24}$. На рис. 2 представлены калибровочные графики этих смесей при различных скоростях программирования температур в колонке. Температуры выхода пз колонки парафинов откладывались против температур кипения (таблица). При дистилляции смесей, выкипающих до $300^{\circ}$, температура в колонке повышалась со скоростью 2 град/мин, тогда как в случае смесей, выкипающих при. более высоких температурах, - 4-10 град/мин.

В основу количественных расчетов хроматографического анализа взят факт, что величина сигналов пламенно-ионизационного детектора пропорциональна весовому содержанию компонентов в смеси. По литературным данным $\left[{ }^{2}\right]$ известно, что ошибка при расчете хроматограмм без применения поправочных коэффициентов для парафино-нафтеновых и ароматических углеводородов остается в пределах $5 \%$.

На рис, 3 представлены кривые разгонки эталонной смеси и неко- 


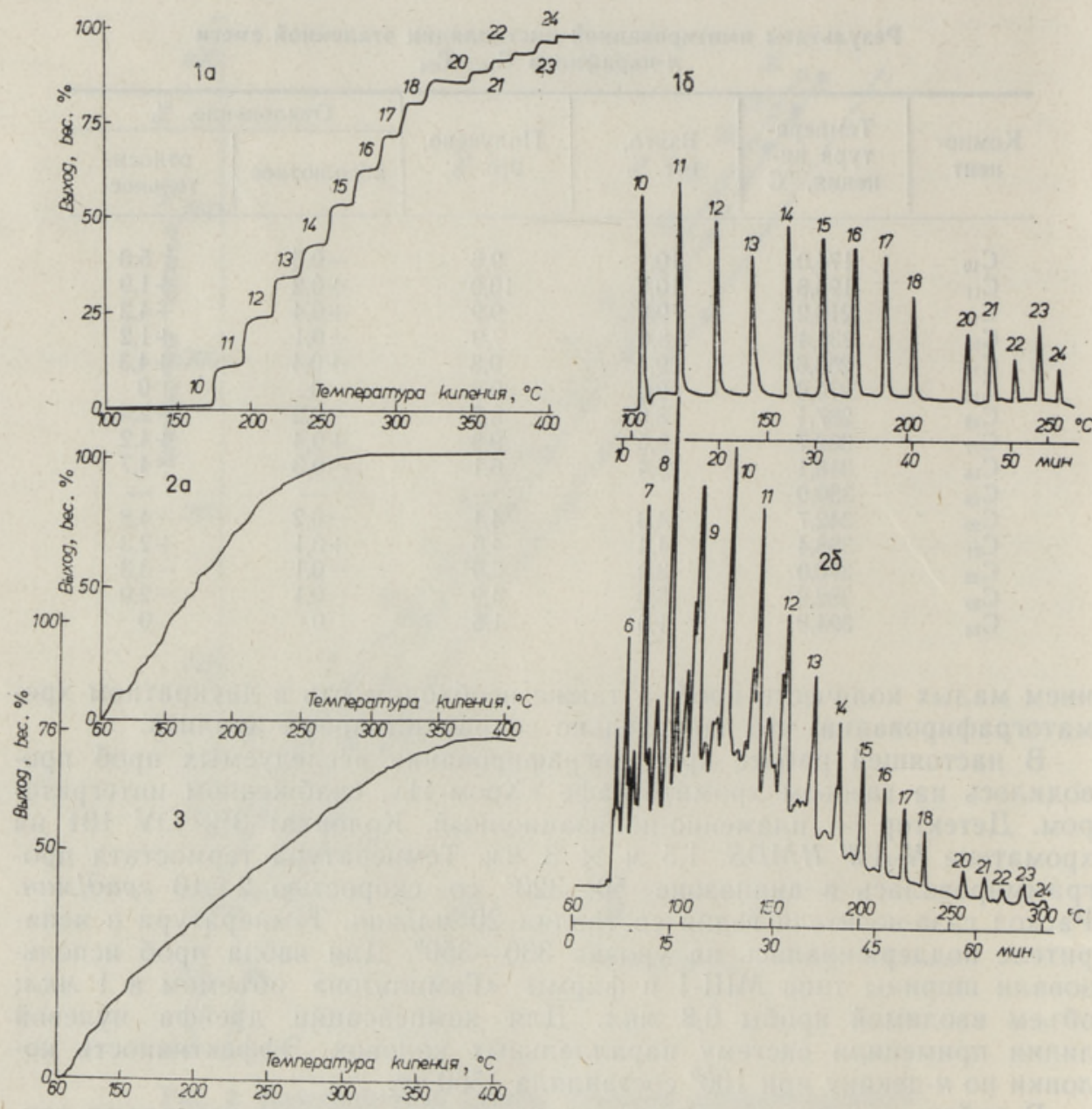

Рис. 3. Кривые разгонки (интегральные хроматограммы $(a)$ ) и дифференциальные хроматограммы (б) эталонной смеси и сланцевых смол. 1 - эталонная смесь $H$-парафинов $\mathrm{C}_{10}-\mathrm{C}_{24}$ (удельный вес 0,7613), 2 - сланцевая смола, 3 - сланцевая смола (удельный вес 0,9201). Цифры на пиках показывают число атомов углерода в молекуле $H$-парафина.

торых сланцевых смол. Для полностью выходящих из колонки смесей, выходы рассчитываются по методу внутренней нормализации: высота ординаты кривой дистилляции отвечает $100 \%$ (рис. $3,2 a$ ). Для смесей же, неполностью выходящих из колонки, при расчетах пользовались данными для эталонной смеси $H$-парафинов $\mathrm{C}_{10}-\mathrm{C}_{24}$, а выход продуктов вычислялся по формуле:

$$
\text { Выход (вес. } \%)=\frac{100 X \vartheta_{c}}{E \vartheta_{x}},
$$

где $E$ - высота ординаты хроматограммы эталонной смеси (рис. 3, 1a), $X$ - высота ординаты хроматограммы исследуемой смеси (рис. $3,3), \partial_{c}-$ удельный вес эталонной смеси, $\partial_{x}$ - удельный вес исследуемой смеси.

Точность результатов, полученных на основе данңых интегральцной 
хроматограммы имитированной дистилляции эталонной смеси, можно оценить по таблице. Как видно, ошибка анализа остается в пределах $5 \%$.

Результаты проведенных нами параллельных опытов с вышеприведенной точностью воспроизводимы для смол с удельными весами до 0,960 г/c ${ }^{3}$. Более вязкие смолы, из которых более $50 \%$ не выходит из колонки, поддаются дозированию по объему с большим трудом. Дистилляционные кривые для смол с удельным весом до 0,960 г/c ${ }^{3}$ характеризуют фракционный состав смол с температурами кипения до $400^{\circ}$.

Описанный метод позволяет определить также количество неотдистиллированной части смолы.

По температурам кипения на кривой дистилляции можно установить также длину цепей $\boldsymbol{H}$-парафинов до $\mathrm{C}_{24}$.

\section{ЛИТЕ РА Т У А}

1. В игдерга уз М. С. Газовая хроматография как метод исследования нефти. М., 1973 , c. $199-207$.

2. Соколова В. И., Берг Г. А., Шкловский Я. А., Ивченко Е. Г., Кузь м и н В. И., Я ро чки н В. Н. Имитированная дистилляция нефтепродуктов, выкипающих при температурах $50-550^{\circ} \mathrm{C}$. - Химия и технология топлива и масел, 1975 , № 6, с. $47-50$.

3. Wormen, J. C., Green, L. G. Simulated distillation of high boiling petroleum fractions. - Anal. Chem., 1965, v. 37, N 12, p. 1620-1621.

4. Green, L. G. Automated simulated distillation with HP $7600 \mathrm{~A}$ chromatograph system. - Anal. Adv., 1969, v. 2, N 3, p. 2-9.

5. Philyaw, L. E., K r c, A. E., O'N e a l, M. J. Gas chromatographic analysis of samples containing both volatile and nonvolatile organic components. Anal. Chem., 1971, v. 43 , N 6, p. $787-789$.

6. Grow, T. H. Simulated distillation of narrow, high boiling hydrocarbon fractions. - Anal. Chem., 1973, v. 45, N 6, p. 987-989.

7. J a cks o n, B. W., Judges, R. W., P owell, J. L. Boiling range distribution of petroleum with a short capillary column. - J. Chromatogr. Sci., 1976, v. $14, \mathrm{~N} \mathrm{2,} \mathrm{p.} 49-51$.

Институт химии
Академии наук Эстонской ССР

Поступила в редакцию $27 / \mathrm{V} 1980$

Saima SALUSTE, H. LUIK, I. KLESMENT

\section{POLEVKIVIOLIDE FRAKTSIOONKOOSTISE MÅRRAMINE IMITEERITUD DESTILLATSIOONI MEETODIL}

On esitatud kuni $400^{\circ} \mathrm{C}$ keevate õlide fraktsioonkoostise määramise lihtne gaasikromatograafiline meetod, mis põhineb proovi täpse mahu ja erikaalu kasutamisel. Keemistemperatuur on määratud kalibreerimisgraafiku abil, mis on esitatud etalonsegu n-parafiinide $\mathrm{C}_{10}-\mathrm{C}_{26}$ väljumis- ja keemistemperatuuri sõltuvusena.

\section{Saima SALUSTE, H. LUIK, I. KLESMENT}

\section{IDENTIFICATION OF COMPOSITION OF SHALE OILS BY SIMULATED DISTILLATION METHOD}

A simple gas chromatographic method based on the use of precise sample content and specific weight is presented for determining fraction composition of oils boiling up to $400^{\circ} \mathrm{C}$. The boiling point is determined by a calibration graph which is given as being dependent on exit boiling point of $n$-parafíns $\mathrm{C}_{10}-\mathrm{C}_{25}$ of the standard test mixture. 